\title{
Ileal Fistula, CTCAE
}

National Cancer Institute

\section{Source}

National Cancer Institute. Ileal Fistula, CT CAE. NCI Thesaurus. Code C57821.

A disorder characterized by an abnormal communication between the ileum and another organ or anatomic site. 